\title{
Practice reduces suppression in metacontrast and in apparent motion
}

\author{
JOHN H. HOGBEN \\ University of Western Australia, Nedlands, Australia \\ and \\ VINCENT DI LOLLO \\ University of Alberta, Edmonton, Alberta, Canada
}

\begin{abstract}
A target display consisting of eight dots drawn randomly from a nine-dot square matrix was displayed for $10 \mathrm{msec}$ and was followed, after intervals ranging from 0 to $160 \mathrm{msec}$, by either two flanking matrices (metacontrast condition) or one flanking matrix (apparent-motion condition). Observers were required to name the location of the missing dot within the target matrix. Identical U-shaped functions of perceptual suppression of the target matrix were obtained in both paradigms. In both cases, level of suppression decreased rapidly with practice so that performance became virtually unimpaired after five testing sessions. The basis of the practice effects was examined in Experiment 2 and was shown to reflect changes in criterion content.
\end{abstract}

Perception of a brief visual target is impaired if a second stimulus (a mask) is displayed nearby and soon after. In a classic investigation of this effectknown as metacontrast masking - Alpern (1953) employed a rectangular target followed by a pair of rectangles, one to each side. When the stimulus-onset asynchrony (SOA) between target and mask was either very brief or very long, the target was perceived clearly and accurately. At intermediate SOAs, however, perception of the target was impaired. The same effect is obtained if the mask surrounds the target completely, rather than merely flanking it (e.g., Werner, 1935).

Almost invariably, metacontrast masking effects are accompanied by an impression of motion between successive parts of the display. This correlative observation has prompted the conjecture that metacontrast and apparent motion effects may be causally related (Breitmeyer, Love, \& Wepman, 1974; Kahneman, 1967). The relationship between the two phenomena was asserted most clearly by Kahneman (1967), who demonstrated the similarity of their temporal requirements. Kahneman (1967) employed a square target followed in time by either two flanking squares (metacontrast paradigm) or only one flanking square (apparent motion paradigm). He found that severity of masking and quality of apparent motion followed identical time courses. Kahneman (1967) also noted that the appearance of the first stimulus was degraded, not only in the metacontrast

Preparation of this article was supported by Grant A6592 from the Natural Sciences and Engineering Research Council of Canada to Vincent Di Lollo. Requests for reprints should be addressed to Vincent Di Lollo, Department of Psychology, University of Alberta, Edmonton, Alberta, Canada T6G 2E9. paradigm, but also in the apparent motion paradigm. A direct comparison of the suppressive effects of metacontrast and of apparent motion was made by Breitmeyer et al. (1974), who obtained equivalent levels of suppression in the two conditions.

The purpose of the present work was to examine further the communalities between metacontrast and apparent motion by comparing their susceptibility to practice effects. Ventura (1980) found that severity of metacontrast masking decreased dramatically over successive experimental sessions. In view of this result, the question arises as to whether perception of the first stimulus improves with practice in an apparent motion paradigm as well in metacontrast. In the present work, the course of practice effects was examined both in a metacontrast and in an apparent motion paradigm. If the two phenomena share a common basis, it should be expected that they would exhibit similar effects of practice.

\section{EXPERIMENT 1}

\section{Method}

The psychophysical task was a modification of a technique previously developed by Hogben and Di Lollo (1974). The target display consisted of a $3 \times 3$ matrix of points plotted on an oscilloscope. One of the nine points, chosen randomly on each trial, was omitted. The observer's task was to identify the location of the missing point by naming its row and column within the matrix. At a variable interval after the target matrix, a second display was presented. This consisted of either one or two flanking matrices to produce conditions of apparent motion or metacontrast, respectively.

Twenty naive observers (undergraduates, paid for their services) were assigned randomly to two experimental conditions of 10 observers each. All observers underwent preliminary training in the use of the response indicator. Training trials consisted of pre- 
sentations of the target matrix simultaneously with either one or two flanking matrices, as appropriate to the condition. Training terminated when the observer was able to make at least 18 correct responses in a block of 20 trials. Two observers were rejected because they could not meet the criterion after six blocks of training trials; they were replaced by two other observers. Following this preliminary session, each observer served for five experimental sessions of 160 trials each.

Stimuli were displayed on a Tektronix 602 oscilloscope with fast P15 phosphor, controlled by a PDP-8/L computer. The observer sat in a dark sound-reduced cubicle, and viewed the oscilloscope through a viewing hood from a distance of $50 \mathrm{~cm}$. A display sequence was initiated when the observer pushed a button.

For the metacontrast condition, the target stimulus was a $3 \times 3$ matrix of points, with a point missing from one randomly determined location on each trial. The matrix was $2.5 \mathrm{~mm}$ square, subtending approximately $0.3 \mathrm{deg}$ of visual angle. The masking stimulus was a pair of complete $3 \times 3$ matrices, each the same size as the target stimulus, and separated from it by $2.2 \mathrm{~mm}$. A typical display pattern is illustrated in Figure 1.

Target and masking stimuli were each of $10 \mathrm{msec}$ duration, and equal in intensity, which was constant for all observers and in all sessions. The whole display was displaced at random from trial to trial, so that the center of the target matrix was either $7.5 \mathrm{~mm}$ above or $7.5 \mathrm{~mm}$ below a central fixation point.

The display for the apparent-motion condition was identical in all respects to the metacontrast display, except that on every trial only one of the flanking matrices (either left or right at random) was presented.

In each condition, the observer's task was to identify the target stimulus by naming the row and column coordinates of the missing point.

Eight levels of stimulus-onset asynchrony (SOA) were employed in each condition. These were 10,20,30,50,80,110, 140, and 170 msec. Twenty trials at each SOA were run, in random order, within an experimental session.

\section{Results}

Mean results across all observers are shown in Figures 2 and 3, for metacontrast and apparent motion, respectively. The group results are representative of the performance of individual observers.

At the end of the experiment, the observers were questioned as to the appearance of the displays and

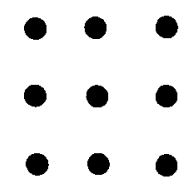

FLANKING
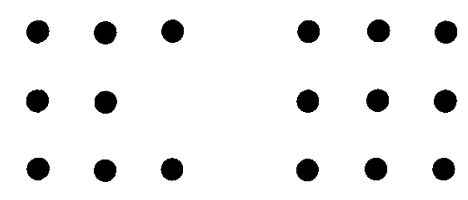

FLANKING

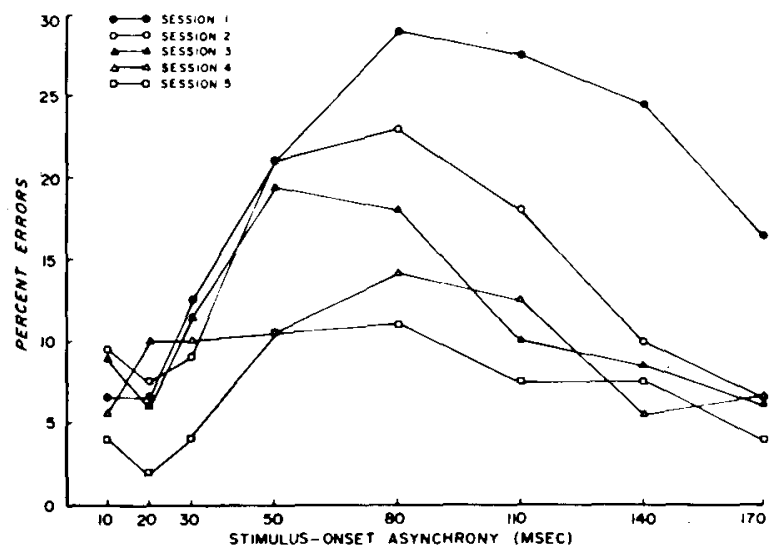

Figure 2. Identification errors as a function of SOA in the metacontrast condition of Experiment 1. Results are shown over five practice sessions.

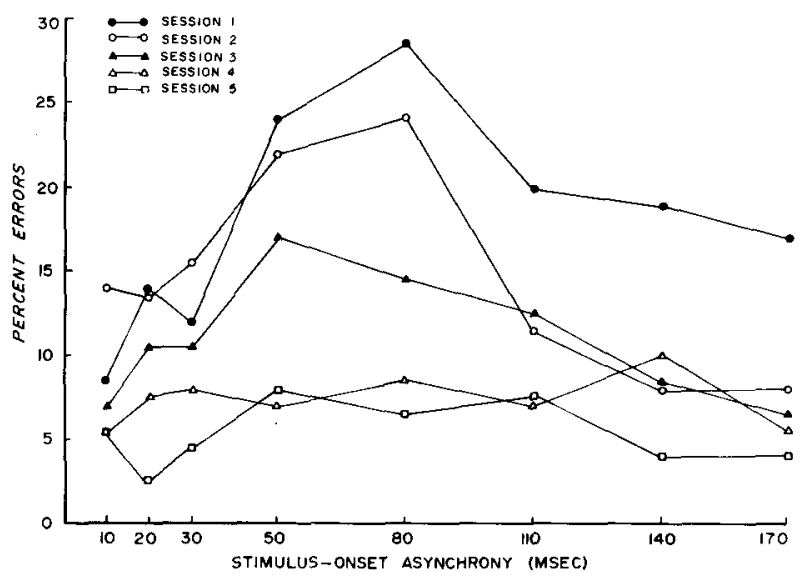

Figure 3. Identification errors as a function of SOA in the apparent-motion condition of Experiment 1. Results are shown over five practice sessions.

as to the basis on which they performed the task. At the briefest SOA, the matrices in each display sequence were seen simultaneously, side by side, with no evidence of motion. At the longest SOAs there was a clear experience that two distinct events had occurred, but there was only a weak impression of motion between successive stimuli. At the middle range of SOAs, there was a strong impression of motion in both conditions. For the metacontrast condition, the impression was of a single matrix giving rise to a pair, each flying in opposite directions. In the "apparent motion" condition, there was an impression that a single matrix had been presented and that it moved across the display surface. These phenomenological reports agree well with common observations in the literature. In particular, the quality of motion generated by the "apparent motion" displays was optimal at intermediate SOAs. This also
Figure 1. Diagrammatic representation of a metacontrast display. The three matrices were shown either above or below fixation, at random. 
is a common finding in the literature (e.g., Kahneman, 1967).

Suppression of the target stimulus under conditions of metacontrast and of apparent motion exhibits very similar inverted U-shaped functions. These results are consistent with the findings of Kahneman (1967), Breitmeyer et al. (1974), and of Breitmeyer, Battaglia, and Weber (1976). Moreover, the effects of practice are the same in the metacontrast and the apparent motion paradigms. Improvement with practice is most evident at intermediate SOAs, at which the suppression effects were initially strongest in both paradigms.

A three-factor analysis of variance performed on the results illustrated in Figures 2 and 3 examined the effects of the two paradigms (metacontrast vs. apparent motion), eight levels of SOA, and five successive practice sessions. The only significant effects were SOA $[F(7,126)=15.14, p<.001]$, practice $[F(4,72)=20.66, p<.001]$, and the interaction effect between SOA and practice $[\mathrm{F}(28,504)=3.90, \mathrm{p}<$ $.001]$. The $F$ ratios for all other effects, namely all effects involving comparisons between paradigms, approximated unity and failed to approach statistical significance.

\section{Discussion}

Powerful and notably similar practice effects were obtained in both the metacontrast and the apparent motion paradigms. In both cases, the results of the first experimental session yielded inverted U-shaped curves showing maximum suppression of the first stimulus at an SOA of 80 msec. Figures 2 and 3 show a steady improvement with practice until, by the fifth experimental session, performance became virtually errorless in both conditions.

These findings parallel those obtained by Ventura (1980), who employed a brightness-estimation task in a metacontrast paradigm. Ventura's findings are extended here in two ways: First, it is shown that practice effects are obtained in apparent motion displays as well as in metacontrast; and, second, it is shown that practice effects also occur with a task requiring identification of the target stimulus.

In Ventura's (1980) work, a briefly displayed vertical bar (the target) was followed by two similar flanking bars (the mask). The observers' task was to rate the brightness of the target over a range of SOAs. The phenomenological appearance of the target was found to be biphasic: an "initial brighter perception of the target was rapidly replaced by a longer dimmer perception when the flanks appeared" (Ventura, 1980, p. 47).

The biphasic appearance of the displays led Ventura to offer an explanation of practice effects in terms of changes in criterion content (Kahneman, 1968). He surmised that early in training observers based their judgments on the appearance of the target in the longer, dimmer phase. However, as training progressed, observers switched their basis of judgment to the brighter initial phase. As a consequence, mean judgments of brightness increased progressively over successive sessions.

Similar changes in criterion content could account for the present results. Namely, if the brightness of the target followed a biphasic time course in our study as it did in Ventura's (1980), the improvement in performance with practice could have resulted from the observers' increasing ability to attend to the brighter initial phase.

But another change in criterion content was suggested by the reports of our observers. On trials in which perception of the target was difficult, one point in the second matrix, or in one of the flanking matrices, would appear to be dimmer or different in some way from its companions. Nominating this point as missing in the target matrix produced a high success rate. This alteration in the appearance of a single point in the mask may be related to the plastic distortions described by Kolers (1972) in apparentmotion paradigms when the two stimuli are similar but not identical. Stewart and Purcell (1970) report a similar phenomenon in a metacontrast paradigm. The mask (a surrounding annulus) appeared to have a missing segment corresponding to the open portion of the previously presented test stimulus, which consisted of the letter $\mathrm{C}$ in different orientations.

It is likely that our observers learned to utilize this cue gradually over successive sessions and that performance improved as a consequence. Experiment 2 was designed to examine this hypothesis.

\section{EXPERIMENT 2}

The strategy adopted in Experiment 2 was aimed at reducing the validity of the plastic transformation in the mask as a cue to the missing element in the target. It was reasoned that if the practice effect were based on this cue, invalidation of the cue should reduce or abolish the practice effect. To achieve this goal, Experiment 2 employed the same apparentmotion paradigm as in Experiment 1, but with a randomly chosen element omitted from the trailing matrix. The missing element in the second matrix was intended to provide a distractor that would interfere with the salience of the cue, and thus forestall the change in criterion content.

\section{Method}

The method was the same as in the apparent motion condition of Experiment 1, except that one of the points in the trailing matrix was eliminated randomly on each trial. No restrictions were placed on the location of the missing point in the trailing matrix, so on about $11 \%$ of the trials the location of the missing points were the same in both matrices.

A new set of 10 naive observers was recruited for this experiment. 


\section{Results and Discussion}

Mean results across observers are shown in Figure 4. A two-factor analysis of variance revealed that the main effect of SOA was significant $[F(7,63)=$ $28.37, \mathrm{p}<.001$ ], as was the main effect of practice $[F(4,36)=4.69, p<.005]$. The interaction effect was not significant $[\mathrm{F}(28,252)=0.82]$.

Although performance improved significantly with practice, the extent of improvement was much attenuated in comparison with that illustrated in Figure 3. Clearly, the omission of one point in the trailing matrix set a limit to the amount of improvement that could be achieved with practice. After five sessions, the performance curves still exhibit the U-shaped function typical of the early stages of practice.

This result is entirely consistent with the diminished availability or validity of the plastic transformation cue. It is possible that the "hole" created by the missing point in the trailing matrix could have demanded the observer's attention to a degree that drastically diminished the prominence-and hence the perceptual availability-of the transformational cue. It is also possible that in Experiment 2 the major plastic transformation consisted of a perceptual migration of the "hole" to the location specified by the trailing matrix. Such a transformation would provide an obviously invalid cue.

Plastic transformations are clearly implicated in the present work as the basis for practice effects. This explanation differs from Ventura's (1980) account in terms of biphasic brightness responses. The two, however, should be seen as complementary rather than contradictory. They are both examples of changes in criterion content, but in different situations. Bi-

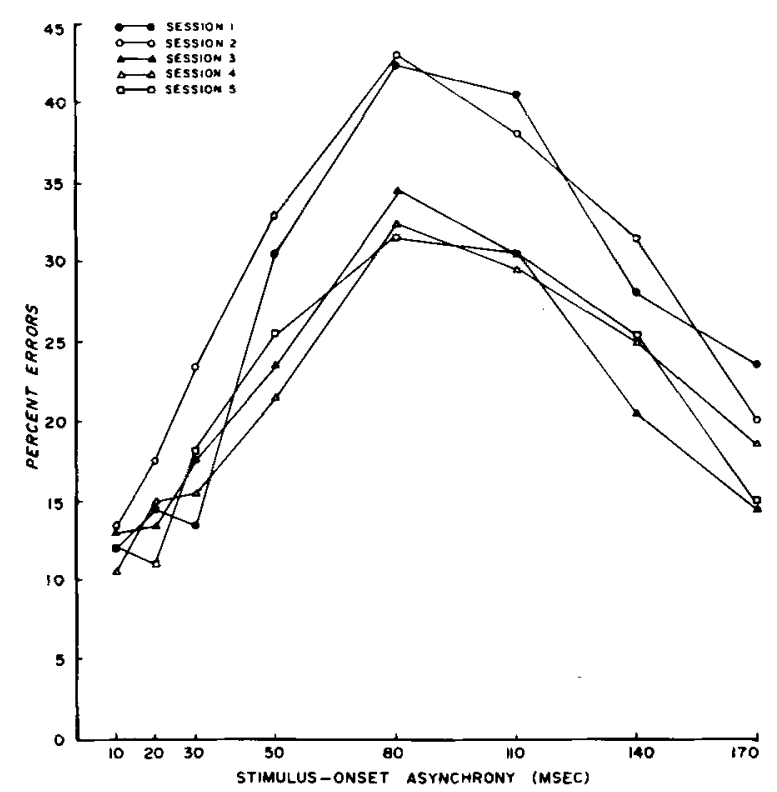

Figure 4. Identification errors as a function of SOA in Experiment 2. Results are shown over five practice sessions. phasic brightness responses are more likely to occurand to be important-in brightness estimation tasks, whereas plastic transformations are likely to be called into play in displays with strong figural properties.

\section{METACONTRAST AND APPARENT MOTION}

In the present work, as well as in that of Breitmeyer et al. (1974), the results obtained with the metacontrast and with the apparent motion paradigms were the same. In addition, our work shows that the progressive improvement in performance with practice followed similar time courses. These facts argue compellingly that suppressive effects in the two paradigms are produced by a common mechanism.

What is the nature of the mechanism of suppression? In seeking an answer to this question, it is appropriate to examine some hypotheses that have been advanced separately to account for suppressive effects in metacontrast and in apparent motion.

Kahneman (1967) explained metacontrast suppression as a case of impossible motion: when an object is made to appear to move in two directions at once, the perceptual system deals with the logical impossibility by suppressing the first stimulus. However, for obvious reasons, impossible motion cannot explain the suppression obtained in the apparent motion paradigm (Figure 3), in which strong coherent motion was perceived. If a unitary explanation is to be sought, it must lie elsewhere.

It is almost a truism that some form of inhibition must play a part in suppressing perception of the first stimulus. Indeed, two major accounts of metacontrast masking have been proposed in terms of lateral inhibition (Weisstein, 1972) and of inhibitory interactions between channels (Breitmeyer \& Ganz, 1976). Whatever its basis, the inhibition is likely to have a significant central component.

Involvement of central loci is suggested by two observations. First, it is known that metacontrast occurs dichoptically (e.g., Kolers \& Rosner, 1960), as does apparent motion (Anstis \& Moulden, 1970; Shipley, Kenney, \& King, 1945); and, second, it has been shown by Uttal (1971) that degree of suppression depends on the structural similarity between target and masking stimuli. This suggests that metacontrast suppression occurs at a processing stage beyond the emergence of pattern. In general terms, the present evidence buttresses Uttal's conclusion that "the metacontrast function is not the result of simple spatial interactions" (Uttal, 1981, p. 728).

A central locus is also compatible with the views expressed by Breitmeyer et al. (1976), who believe that suppressive effects in both metacontrast and apparent motion are in some ways related to the perception of motion itself. They note that motion is perceived in both types of displays and conclude that "contour suppression therefore is a phenomenon at- 
tending perceptually possible and vivid stroboscopic motion"' (Breitmeyer et al., 1976, p. 170).

Whether the phenomenal appearance of motion is necessary for suppression to occur or whether separate mechanisms are at work is not answered in the present study. Appearance of motion and degree of suppression were certainly correlated in our experiments, but this correlative occurrence cannot be considered evidence of causation. Our preference is to suggest that the mechanisms responsible for the sensation of motion and for the suppression of temporally leading stimuli are largely independent. To wit, we suggest that the perception of an object in motion emerges from the activity of largely independent mechanisms, including a motion-signaling system as well as a suppressive system. This hypothesis is compatible with the views of Breitmeyer et al. (1976), and can account for the impression of motion and for the suppressive effects observed in both metacontrast and apparent-motion displays.

It is plausible to assume that ordinarily all subsystems may operate synchronously to produce perception of motion. But it is equally plausible to expect that separate subsystems would respond differently to variations in spatiotemporal patterns of stimulation. And this may well have been the case in Weisstein and Growney's (1969) study, in which optimal apparent motion and maximal metacontrast were shown to have different spatiotemporal parameters.

\section{REFERENCES}

Alpern, M. (1953). Metacontrast. Journal of the Optical Society of America, 43, 648-657.

Anstis, S. M., \& Moulden, B. P. (1970). After effect of seen movement: Evidence for peripheral and central components. Quarterly Journal of Experimental Psychology, 22, 222-229.

Breitmeyer, B., BatTaglia, F., \& Weber, C. (1976). U-shaped backward contour masking during stroboscopic motion. Journal of Experimental Psychology: Human Perception and Performance, 2, 167-173.
Breitmeyer, B. G., \& Ganz, L. (1976). Implications of sustained and transient channels for theories of pattern masking, saccadic suppression, and information processing. Psychological Review, 83, 1-36.

Breitmeyer, B. G., Love, R., \& Wepman, B. (1974). Contour suppression during stroboscopic motion and metacontrast. Vision Research, 14, 1451-1456.

Hogben, J. H., \& Di Lollo, V. (1974). Perceptual integration and perceptual segregation of brief visual stimuli. Vision $R e-$ search, 14, 1059-1069.

KaHNEmAN, D. (1967). An onset-onset law for one case of apparent motion and metacontrast. Perception \& Psychophysics, 2, 577-584.

Kahneman, D. (1968). Method, findings, and theory in studies of visual masking. Psychological Bulletin, 70, 404-425.

Kolers, P. A. (1972). Aspects of motion perception. Oxford: Pergamon Press.

Kole rs, P. A., \& Rosner, B. S. (1960). On visual masking (metacontrast): Dichoptic observation. American Journal of Psychology, 73, 2-21.

Shipley, W. C., Kenney, F. A., \& King, M. E. (1945). Beta apparent movement under binocular, monocular and interocular stimulation. American Journal of Psychology, 58, 545-549.

Stewart, A. L., \& Purcell, D. G. (1970). U-shaped masking functions in visual backward masking: Effects of target configuration and retinal position. Perceptual \& Psychophysics, 7, 253-256.

UTTAL, W. R. (1971). The psychobiological silly season or what happens when neurophysiological data become psychological theories. Journal of General Psychology, 84, 151-166.

UTTAL, W. R. (1981). A taxonomy of visual processes. Hillsdale, NJ: Erlbaum.

Ventura, J. (1980). Foveal metacontrast: I. Criterion content and practice effects. Journal of Experimental Psychology: Human Perception and Performance, 6, 473-485.

Weisstein, N. (1972). Metacontrast. In D. Jameson \& L. M. Hurvich (Eds.), Handbook of sensory physiology (Vol. 7). New York: Springer-Verlag.

Weisstein, N., \& Growney, R. L. (1969). Apparent movement and metacontrast: A note on Kahneman's formulation. Perception \& Psychophysics, 5, 321-328.

We RNER, H. (1935). Studies on contour. I. Qualitative analyses. American Journal of Psychology, 47, 40-64.

(Manuscript received January 6, 1984; revision accepted for publication March 14, 1984.) 